\title{
A Short Commentary on Globus Pallidus Internus Deep Brain Stimulation in Primary Meige Syndrome
}

\section{Ryoma Morigaki $^{1-3}$ and Satoshi Goto ${ }^{1,2^{*}}$}

${ }^{1}$ Parkinson's Disease and Dystonia Research Center, Tokushima University Hospital, Tokushima University, Tokushima, Japan

${ }^{2}$ Department of Neurodegenerative Disorders Research, Institute of Biomedical Sciences, Graduate School of Medical Sciences, Tokushima University, Tokushima, Japan

${ }^{3}$ Department of Neurosurgery, Institute of Biomedical Sciences, Graduate School of Medical Sciences, Tokushima University, Tokushima, Japan

*Corresponding author: Satoshi Goto, Department of Neurodegenerative Disorders Research, Institute of Biomedical Sciences, Graduate School of Medical Sciences, Tokushima University, Tokushima 770-8503, Japan, Tel: +81-88-633-7206; Fax: +81-88-633-7208; E-mail: sgoto@tokushima-u.ac.jp

Received date: November 25, 2016; Accepted date: December 19, 2016; Published date: December 26, 2016

Copyright: ( 2016 Morigaki R, et al. This is an open-access article distributed under the terms of the Creative Commons Attribution License, which permits unrestricted use, distribution, and reproduction in any medium, provided the original author and source are credited.

\begin{abstract}
Meige syndrome is a type of segmental dystonia that manifests a combination of blepharospasm and oromandibular dystonia and is often associated with other types of craniocervical dystonia. Although the precise pathogenesis of primary Meige syndrome remains to be elucidated, it has been suggested that this movement disorder might be a basal ganglia disorder. Multiple single case reports and open-labeled small case series have shown that globus pallidus internus deep brain stimulation (GPi-DBS) could result in therapeutic benefits in patients with severe Meige syndrome. However, randomized and controlled trials are necessary to accurately assess the safety and therapeutic efficacy of GPi-DBS in treating patients with Meige syndrome. This short commentary introduces the current use of GPi-DBS in the treatment of medically refractory Meige syndrome.
\end{abstract}

Keywords: Meige syndrome; Deep brain stimulation; Globus pallidus internus; Blepharospasm; Craniocervical dystonia; Segmental dystonia

\section{Introduction}

Meige syndrome was first described in detail by Henri Meige [1], the French neurologist. This movement disorder is a type of dystonia, and is also known as Bruegel's syndrome [2] and oral facial dystonia. Meige syndrome is characterized by the presence of blepharospasm in combination with oromandibular dystonia and is often associated with other types of craniofacial dystonias [3]. Meige syndrome affects women more often than men (2:1 ratio) and its symptom onset is typically between 30 and 70 years of age. In general, Meige syndrome responds poorly to oral pharmacotherapy [4-6]. A double-blind study [7] has proven that trihexyphenidyl, an antimuscarinic agent, is effective in the treatment of segmental craniocervical dystonias that include Meige syndrome. However, only 1 of 9 patients with Meige syndrome responded to trihexyphenidyl in this study. The United States Food and Drug Administration (FDA) has approved botulinum toxin type A (BTA) as a first-line treatment for blepharospasm and cervical dystonias $[3,8]$. Since blepharospasm associated with Meige syndrome is usually more severe than isolated blepharospasm, it is difficult to obtain satisfactory results with BTA injections in the treatment of Meige syndrome [8-10]. In addition, BTA treatments have the potential to cause adverse events such as diminished responses, facial weakness, dysphagia, dry mouth, flu-like syndrome, and the development of antibodies against botulinum toxin [8,11-13]. Despite these best medical attempts, intractable disabling dystonias often persist in some patients with primary Meige syndrome.

The precise cause of primary Meige syndrome is poorly understood, however it is thought to be a variant of idiopathic torsion dystonia $[14,15]$. As in other dystonia syndromes, multiple single case reports and open-labeled small case series have shown that bilateral deep brain stimulation (DBS) of the globus pallidus internus (GPi), which is the major basal ganglia output nucleus, can produce powerful therapeutic benefits in medically intractable primary Meige syndrome [16]. The therapeutic efficacy of GPi-DBS as determined by Burke-FahnMarsden dystonia rating scale total movement score (BFMDRS-M) showed an improvement of $64.3 \%$ (SD 29.5) [17]. In the selected Meige syndrome patients with longer follow-up periods, that is, for more than 5 years (mean $\pm \mathrm{SD}, 80.7 \pm 21.9$ months), the therapeutic efficacy of GPi-DBS resulted in an improvement of $70.9 \pm 17.1 \%$ (mean \pm SD), as determined by BFMDRS-M [18-21]. Overall clinical outcomes suggest that GPi-DBS might be more efficient in patients with Meige syndrome than in those with other types of focal and segmental dystonias [17]. The beneficial effects of GPi-DBS in patients with Meige syndrome could be maintained for more than 5 years after surgery. However, the true benefits of GPi-DBS are currently unclear due to the lack of randomized controlled trials in patients with Meige syndrome. The stereotactic coordinates for active contacts of the DBS lead usually are usually located in the posteroventrolateral portion of the GPi [22-26]. Stimulation parameters used in patients with primary Meige syndrome were highly variable with amplitude of 1.0-5.2 volts, frequency of 60$235 \mathrm{~Hz}$, and pulse width of 90-500 $\mu$ s [16,18-29]. In our experience, a long pulse width (e.g. $450 \mu \mathrm{s})$ usually yielded good clinical results $[21,27,28]$. Using the meta-regression analysis on patients with dystonias that include Meige syndrome, Andrews et al. reported that the only variable showing a trend towards association with improved percentage outcome by GPi-DBS was a shorter time duration between the disease onset and DBS initiation $(\mathrm{p}=0.06)$ [17].

GPi-DBS should be considered only if the patients experienced markedly disabling motor symptoms associated with Meige syndrome even though they had received other treatments options, administered by skilled and experienced neurologists [3]. The exclusion criteria for the use of GPi-DBS in patients with Meige syndrome are essentially the 
same as those applied to patients with other primary dystonias. They include the presence of brain atrophy and/or other organic lesions, marked cognitive impairment, acute psychiatric changes, severe depression, and other coexisting medical disorders that would increase surgical risk [30]. Perioral tightness [18] and stimulation-induced severe parkinsonism [29] have been reported as un-adjustable complications caused by GPi-DBS in patients with Meige syndrome. Ostrem et al. reported difficulty with coordination and slowness in motor function including worsening of handwriting, typing, balance, and walking in 4 of 6 patients with Meige syndrome [22]. We also should pay a careful attention to depression, which can be a potential risk factor for suicide after GPi-DBS [31]. The parallel organization of functionally segregated cortico-basal ganglia-thalamo-cortical feedback loops invokes the possibility that GPi-DBS could influence motor as well as the other functional loops including associative or limbic loops [32,33]. Ventral two-thirds of the posterior GPi is the primary motor cortex-related territory and dorsal one-third of the posterior GPi is the prefrontal cortex-related territory [34,35]. More dorsal and anterior to the motor cortex-related territory is the supplementary motor area-related territory, while the most medial part of the GPi corresponds to the limbic cortex-related territory [34,35]. For those reason, correct positioning of the active electrodes and careful follow-up assessments of non-motor symptoms including psychiatric conditions after GPi-DBS should be required. In summary, bilateral GPi-DBS could produce a significant improvement of medically refractory dystonia symptoms in patients with Meige syndrome. In the near future, well-designed studies with randomized and controlled trials will be required to establish GPi-DBS as an effective and tolerable treatment option for Meige syndrome.

\section{Conflict of Interest Statement}

The authors declare that the research was conducted in the absence of any commercial or financial relationships that could be construed as a potential conflict of interest.

\section{Acknowledgement}

This work was supported in part by grants from the Ministry of Education, Culture, Sports, Science, and Technology of Japan (grantsin-aid for Scientific Research no. 24390223, 26461272, 26430054, and 16k10788), and Japan Agency for Medical Research and Development (AMED) (no. 16ek0109182h0001).

\section{References}

1. Meige H (1910) Les convulsions de la face, une forme clinique deconvulsion faciale, bilatérale et médiane. Revista de Neurología 20: 437-443.

2. Marsden CD (1976) Blepharospasm-oromandibular dystonia syndrome (Brueghel's syndrome). A variant of adult-onset torsion dystonia? J Neurol Neurosurg Psychiatry 39: 1204-1209.

3. LeDoux MS (2009) Meige syndrome: what's in a name? Parkinsonism Relat Disord 15: 483-489.

4. Jankovic J, Orman J (1984) Blepharospasm: demographic and clinical survey of 250 patients. Ann Ophthalmol 16: 371-376.

5. Grandas F, Elston J, Quinn N, Marsden CD (1988) Blepharospasm: a review of 264 patients. J Neurol Neurosurg Psychiatry 51: 767-772.

6. Castelbuono A, Miller NR (1998) Spontaneous remission in patients with essential blepharospasm and Meige syndrome. Am J Ophthalmol 126: 432-435.
7. Nutt JG, Hammerstad JP, deGarmo P, Carter J (1984) Cranial dystonia: double-blind crossover study of anticholinergics. Neurology 34: 215-217.

8. Defazio G, Livrea P (2004) Primary blepharospasm: diagnosis and management. Drugs 64: 237-244.

9. Mauriello JA Jr, Dhillon S, Leone T, Pakeman B, Mostafavi R, et al. (1996) Treatment selections of 239 patients with blepharospasm and Meige syndrome over 11 years. Br J Ophthalmol 80: 1073-1076.

10. Bhidayasiri R, Cardoso F, Truong DD (2006) Botulinum toxin in blepharospasm and oromandibular dystonia: comparing different botulinum toxin preparations. Eur J Neurol 13 Suppl 1: 21-29.

11. Jankovic J (2004) Treatment of cervical dystonia with botulinum toxin. Mov Disord 19 Suppl 8: S109-115.

12. Jost WH, Kohl A (2001) Botulinum toxin: evidence-based medicine criteria in blepharospasm and hemifacial spasm. J Neurol 248: 21-24.

13. Dressler D, Benecke R (2003) Autonomic side effects of botulinum toxin type B treatment of cervical dystonia and hyperhidrosis. Eur Neurol 49: 34-38.

14. Marsden CD, Sheehy MP (1982) Spastic dysphonia, Meige disease, and torsion dystonia. Neurology 32: 1202-1203.

15. Berardelli A, Rothwell JC, Day BL, Marsden CD (1985) Pathophysiology of blepharospasm and oromandibular dystonia. Brain 108: 593-608.

16. Wang X, Zhang C, Wang Y, Liu C, Zhao B, et al. (2016) Deep brain stimulation for craniocervical dystonia (Meige syndrome): a report of four patients and a literature-based analysis of its treatment effects. Neuromodulation 19: 818-823.

17. Andrews C, Aviles-Olmos I, Hariz M, Foltynie T (2010) Which patients with dystonia benefit from deep brain stimulation? A metaregression of individual patient outcomes. J Neurol Neurosurg Psychiatry 81: 1383-1389.

18. Loher TJ, Capelle HH, Weber S, Weigel R, Burgunder JM, et al. (2007) Deep brain stimulation for dystonia: outcome at long-term follow-up. J Neurol 255: 881-884.

19. Reese R, Gruber D, Schoenecker T, Bäzner H, Blahak C, et al. (2011) Long-term clinical outcome in Meige syndrome treated with internal pallidum deep brain stimulation. Mov Disord 26: 691-698.

20. Tai CH, Wu RM, Liu HM, Tsai CW, Tseng SH (2011) Meige syndrome relieved by bilateral pallidal stimulation with cycling mode: case report. Neurosurgery 69: E1333-1337.

21. Sako W, Morigaki R, Mizobuchi Y, Tsuzuki T, Ima H, et al. (2011) Bilateral pallidal deep brain stimulation in primary Meige syndrome. Parkinsonism Relat Disord 17: 123-125.

22. Ostrem JL, Marks WJ Jr, Volz MM, Heath SL, Starr PA (2007) Pallidal deep brain stimulation in patients with cranial-cervical dystonia (Meige syndrome). Mov Disord 22: 1885-1891.

23. Hebb MO, Chiasson P, Lang AE, Brownstone RM, Mendez I (2007) Sustained relief of dystonia following cessation of deep brain stimulation. Mov Disord 22: 1958-1962.

24. Blomstedt P, Tisch S, Hariz MI (2008) Pallidal deep brain stimulation in the treatment of Meige syndrome. Acta Neurol Scand 118: 198-202.

25. Markaki E, Kefalopoulou Z, Georgiopoulos M, Paschali A, Constantoyannis C (2009) Meige's syndrome: a cranial dystonia treated with bilateral pallidal deep brain stimulation. Clin Neurol Neurosurg 112: 334-346.

26. Ghang JY, Lee MK, Jun SM, Ghang CG (2010) Outcome of pallidal deep brain stimulation in meige syndrome. J Korean Neurosurg Soc 48: 134-138.

27. Muta D, Goto S, Nishikawa S, Hamasaki T, Ushio Y, et al. (2001) Bilateral pallidal stimulation for idiopathic segmental axial dystonia advanced from Meige syndrome refractory to bilateral thalamotomy. Mov Disord 16:774-777.

28. Inoue N, Nagahiro S, Kaji R, Goto S (2010) Long-term suppression of Meige syndrome after pallidal stimulation: a 10-year follow-up study. Mov Disord 25: 1756-1758.

29. Zauber SE, Watson N, Comella CL, Bakay RA, Metman LV (2009) Stimulation-induced parkinsonism after posteroventral deep brain 
Citation: Morigaki R, Goto S (2016) A Short Commentary on Globus Pallidus Internus Deep Brain Stimulation in Primary Meige Syndrome. J Neurol Neurophysiol 7: 405. doi:10.4172/2155-9562.1000405

Page 3 of 3

stimulation of the globus pallidus internus for craniocervical dystonia. J Neurosurg 110: 229-233.

30. Kupsch A, Benecke R, Müller J, Trottenberg T, Schneider GH, et al. (2006) Pallidal deep-brain stimulation in primary generalized or segmental dystonia. N Engl J Med 355: 1978-1990.

31. Foncke EM, Schuurman PR, Speelman JD (2006) Suicide after deep brain stimulation of the internal globus pallidus for dystonia. Neurology 66: 142-143.

32. Alexander GE, DeLong MR, Strick PL (1986) Parallel organization of functionally regregated circuits linking basal ganglia and cortex. Annu Rev Neurosci 9: 357-381.
33. Sheline YI (2003) Neuroimaging studies of mood disorder effects on the brain. Biol Psychiatry 54: 338-352.

34. Nambu A (2011) Somatotopic organization of the primate basal ganglia. Front Neuroanat 5: 26.

35. Morigaki R, Mure H, Nagahiro S, Kaji R, Goto S (2016) Therapeutic perspective on tardive syndrome with special reference to deep brain stimulation. Front Psychiatry 7: 207. 